\title{
CN Com: a Typical RS Canum Venaticorum Star
}

\author{
G.A. Richter ${ }^{1}$, J. Greiner ${ }^{2}$ \\ 1 Sternwarte Sonneberg, 96515 Sonneberg, Germany \\ 2 Max-Planck-Institut für Extraterrestrische Physik, 85740 Garching, Germany
}

CN Com = CSV 6907 was dicovered by Romano (1958). First we note that the GCVS (and Simbad) coordinates are wrong by $\approx 2^{\prime}$. Using the finding chart of Romano (1958) and the APM data (digitized POSS), the correct coordinates of CN Com are R.A. $(2000.0)=12^{\mathrm{h}} 19^{\mathrm{m}} 47^{\mathrm{s}} 0, \operatorname{DEC}(2000.0)=+16^{\circ} 30^{\prime} 50^{\prime \prime}$.

CN Com is only $8^{\prime \prime}$ distant from the ROSAT X-ray source RX J1219.7+1630 detected during the All-Sky-Survey at a PSPC countrate of $0.022 \mathrm{cts} / \mathrm{s}$. Due to the positional proximity and the absence of other optical objects brighter than $20^{r m m}$ within the about $30^{\prime \prime}$ error circle, CN Com is very probably the optical counterpart of RX J1219.7+1630.

CN Com was investigated on some 600 photographic plates (Sonneberg astrographs $400 / 1600 \mathrm{~mm}$ and $400 / 2000 \mathrm{~mm}$ ) of the fields $26 \mathrm{Com}$ and $5 \mathrm{Com}$, covering the time interval from 1962 to 1994 (with only a very few plates from the years 1967-1974).

The elements are:

$$
T(\min )=2437668.521+0.7354422 \cdot E,
$$

which deviate only negligibly from the dates given by Meinunger (1968).

Figure 1 gives the year by year light curves (from January to June, each). One can see migrating waves typical for RS Canum Venaticorum stars. Sometimes the amplitude of the primary minimum is very small (only $0^{\mathrm{m}} 3$ in 1963,1975 , $1982)$, at other times large $(0.7$ in $1962,1984,1985,1991)$. In some years the amplitude is unknown because there are no observations near phase $0(1965$, 1976-1980, 1983, 1986-1988, 1993/1994). In these cases, other plate collections should be checked. Probably it is not merely the primary that is eclipsed but rather a disk or a halo of varying luminosity and size.

Acknowledgement: This work has been supported by funds of the German Bundesministerium für Forschung und Technologie under grant 05 2SO524 (GAR), and FKZ 50 OR 9201 (JG). We are grateful to R. McMahon for installing the software used for the APM finding charts. The ROSAT project is supported by the German Bundesministerium für Forschung und Technologie (BMFT/DARA) and the Max Planck Society. 


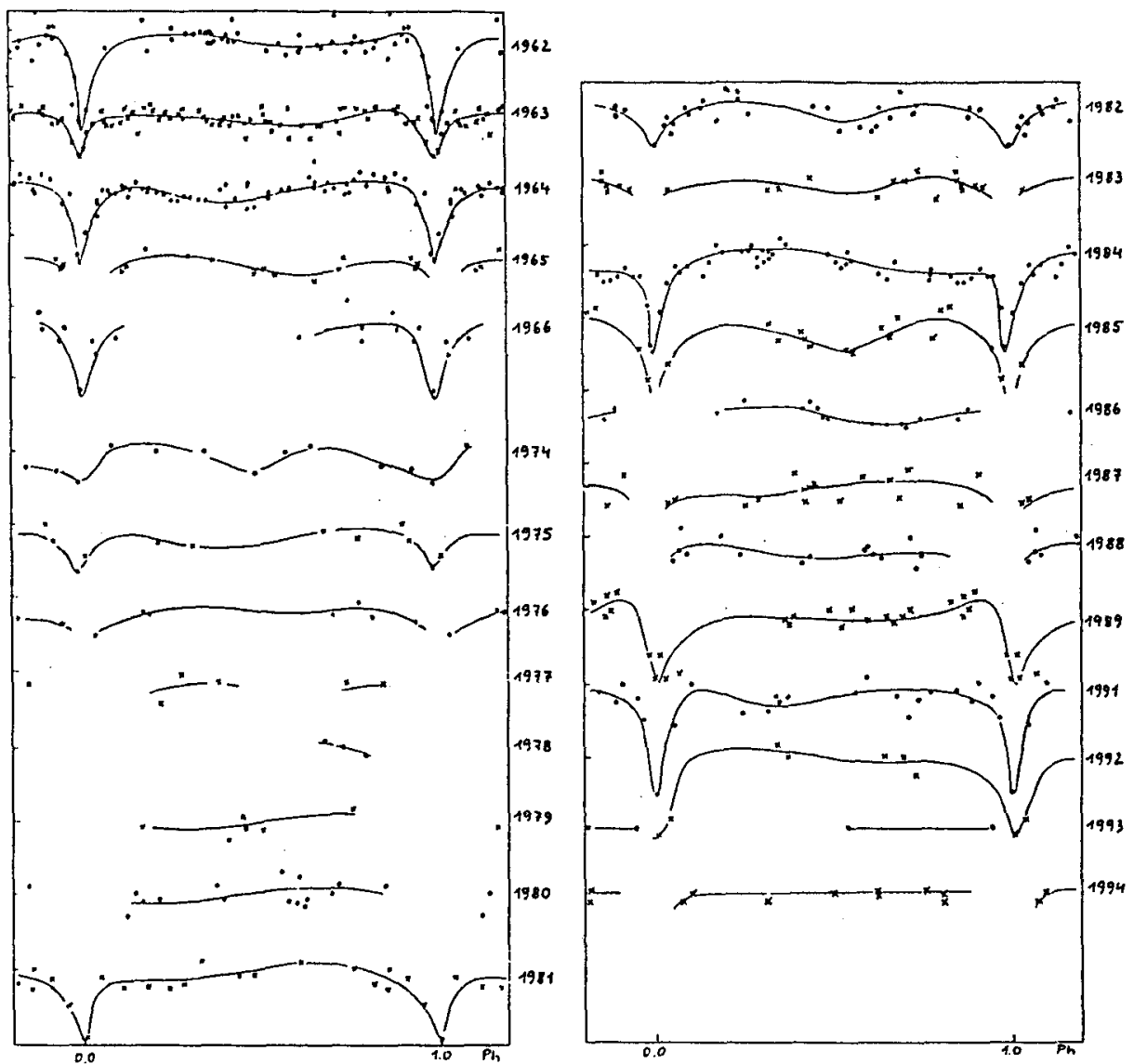

Fig. 1. Light curve of CN Com as deduced from the astrographic plates (field patrol) of Sonneberg Observatory. The migrating waves clearly classify $\mathrm{CN}$ Com as a typical RS CVn star.

\section{References}

Meinunger L., 1968, Veröff. Sternw. Sonneberg 7, 385

Romano G., 1958, Coelum 26, No. 108 